Supplement of Biogeosciences, 15, 1895-1918, 2018

https://doi.org/10.5194/bg-15-1895-2018-supplement

(C) Author(s) 2018. This work is distributed under

the Creative Commons Attribution 3.0 License.

(c) (1)

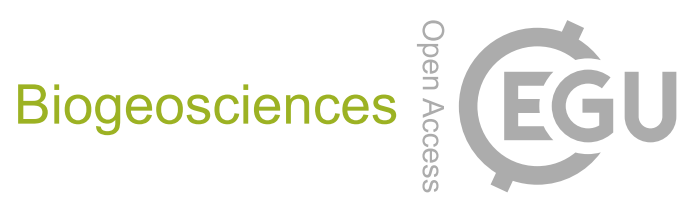

Supplement of

\title{
Biological production in the Indian Ocean upwelling zones - Part 1: refined estimation via the use of a variable compensation depth in ocean carbon models
}

Mohanan Geethalekshmi Sreeush et al.

Correspondence to: Mohanan Geethalekshmi Sreeush (sreeushmg@tropmet.res.in)

The copyright of individual parts of the supplement might differ from the CC BY 3.0 License. 


\section{Sensitivity Simulations}

From the analysis of four major upwelling regions over the Indian Ocean, it is evident that the biological parameterization of varZc better captures upwelling episodes and thus it enhances the model export production. This is most clearly visible over the WAS region. In order to quantify how much the varZc parameterization contributes to the seasonality of carbon cycle, two additional sensitivity simulations are carried out; viz. (1) with annual mean offline currents and (2) annual mean offline temperatures with the goal of suppressing the dynamical and thermodynamical effects of seasonal upwelling over WAS (see Section 2 for details). The focus on this region is motivated by its prominence as the most productive zone of the Indian Ocean. Moreover, the improvement in the biological processes in the model by the varZc parameterization is best captured in this region. The results are discussed below.

\subsection{Impact of varZc parameterization on seasonality of carbon cycle with annual mean currents.}

To quantify the impact of varZc parameterization, the model is forced with annual mean currents only over WAS region with unaltered currents in the rest of the ocean. The hypothesis is that the muting of the seasonal variability of Ekman divergence removes the upwelling and the biological pump contribution to production and carbon cycle. The comparison of constZc and varZc then allows us to decipher the impact of varZc in capturing the impacts of upwelling on biological production and the carbon cycle.

The results indicate that varZc parameterization is responsible for an improvement of $0.48 \pm$

$0.04 \mathrm{~mol} \mathrm{~m}^{-2} \mathrm{yr}^{-1}$ and $0.13 \pm 0.02 \mathrm{~mol} \mathrm{~m}^{-2} \mathrm{yr}^{-1}$ in the JJAS seasonal and annual mean $\mathrm{CO}_{2}$ fluxes, 
respectively. This improves the overall model $\mathrm{CO}_{2}$ flux in the control run especially in July (Figure S1(b)). Similar improvements are also noticed in $\mathrm{pCO}_{2}$ (Figure S2). The improvements are also obvious in export and new production (see Table S1).

In short the varZc biological parameterization improves the export and new productions in the model. This helps the model to capture the upwelling episodes over the study regions. Table S1 summarizes all the results of biological sensitivity runs.

\subsection{Impact of varZc parameterization on seasonality of carbon cycle with annual mean temperatures.}

By imposing the annual mean temperature over WAS region, we are suppressing the cooling effect of temperature due to upwelling and quantifying how much the model seasonality is improved due to varZc parameterization. (see Section 2 for details). The varZc simulations

forced with annual mean SST has larger JJAS mean and annual mean $\mathrm{CO}_{2}$ fluxes with a dominant control by the solubility pump when varZc is employed. The detailed quantification of $\mathrm{CO}_{2}$ and $\mathrm{pCO}_{2}$ responses for this experimental setup is given in Table $\mathrm{S} 2$. The above analysis adds supporting evidence that the varZc simulation strengthens the seasonality of the model compared to the constZc case. This is presumably accomplished by the more accurate $\mathrm{Zc}$ and production zone implied with a variable Zc. 
Table S1: JJAS mean and climatological annual mean response from the model forced with annual mean currents.

\begin{tabular}{|c|c|c|c|c|c|c|}
\hline & \multicolumn{6}{|c|}{ WAS region forced with Annual Mean Currents } \\
\hline & \multicolumn{3}{|c|}{$\underline{\text { JJAS mean }}$} & \multicolumn{3}{|c|}{ Climatological annual mean } \\
\hline & constZc & $\operatorname{varZc}$ & Improvement & constZc & varZc & Improvement \\
\hline $\mathrm{CO}_{2}$ flux $\left(\mathrm{mol} \mathrm{m}^{-2} \mathrm{yr}^{-1}\right)$ & $0.80 \pm 0.2$ & $1.29 \pm 0.2$ & $0.48 \pm 0.04$ & $0.65 \pm 0.1$ & $0.79 \pm 0.1$ & $0.13 \pm 0.02$ \\
\hline $\mathrm{pCO}_{2}(\mu \mathrm{atm})$ & $381.81 \pm 3.4$ & $387.24 \pm 3.9$ & $5.43 \pm 0.5$ & $388.68 \pm 3.4$ & $388.40 \pm 3.6$ & $-0.28 \pm 0.1$ \\
\hline Export production $\left(\mathrm{g} \mathrm{C} \mathrm{m}^{-2} \mathrm{yr}^{-1}\right)$ & $60.71 \pm 4.7$ & $104.22 \pm 13.4$ & $43.51 \pm 8.6$ & $74.30 \pm 4.5$ & $104.58 \pm 18.3$ & $30.28 \pm 13.7$ \\
\hline New Production $\left(\mathrm{g} \mathrm{C} \mathrm{m}^{-2} \mathrm{yr}^{-1}\right)$ & $34.76 \pm 2.3$ & $52.16 \pm 1.51$ & $17.39 \pm 0.8$ & $29.91 \pm 1.7$ & $44.72 \pm 1.6$ & $14.81 \pm 0.1$ \\
\hline
\end{tabular}

Table S2: Same as Table S1 but from annual mean temperature simulation.

\begin{tabular}{|c|c|c|c|c|c|c|}
\hline & \multicolumn{6}{|c|}{ WAS region forced with Annual mean temperature } \\
\hline & \multicolumn{3}{|c|}{ JJAS mean } & \multicolumn{3}{|c|}{ Climatological annual mean } \\
\hline & constZc & varZc & Improvement & constZc & varZc & Improvement \\
\hline $\mathrm{pCO}_{2}(\mu \mathrm{atm})$ & $393.20 \pm 3.01$ & $404.26 \pm 4.9$ & $11.05 \pm 1.9$ & $384.61 \pm 3.3$ & $386.52 \pm 4.8$ & $1.91 \pm 1.4$ \\
\hline
\end{tabular}




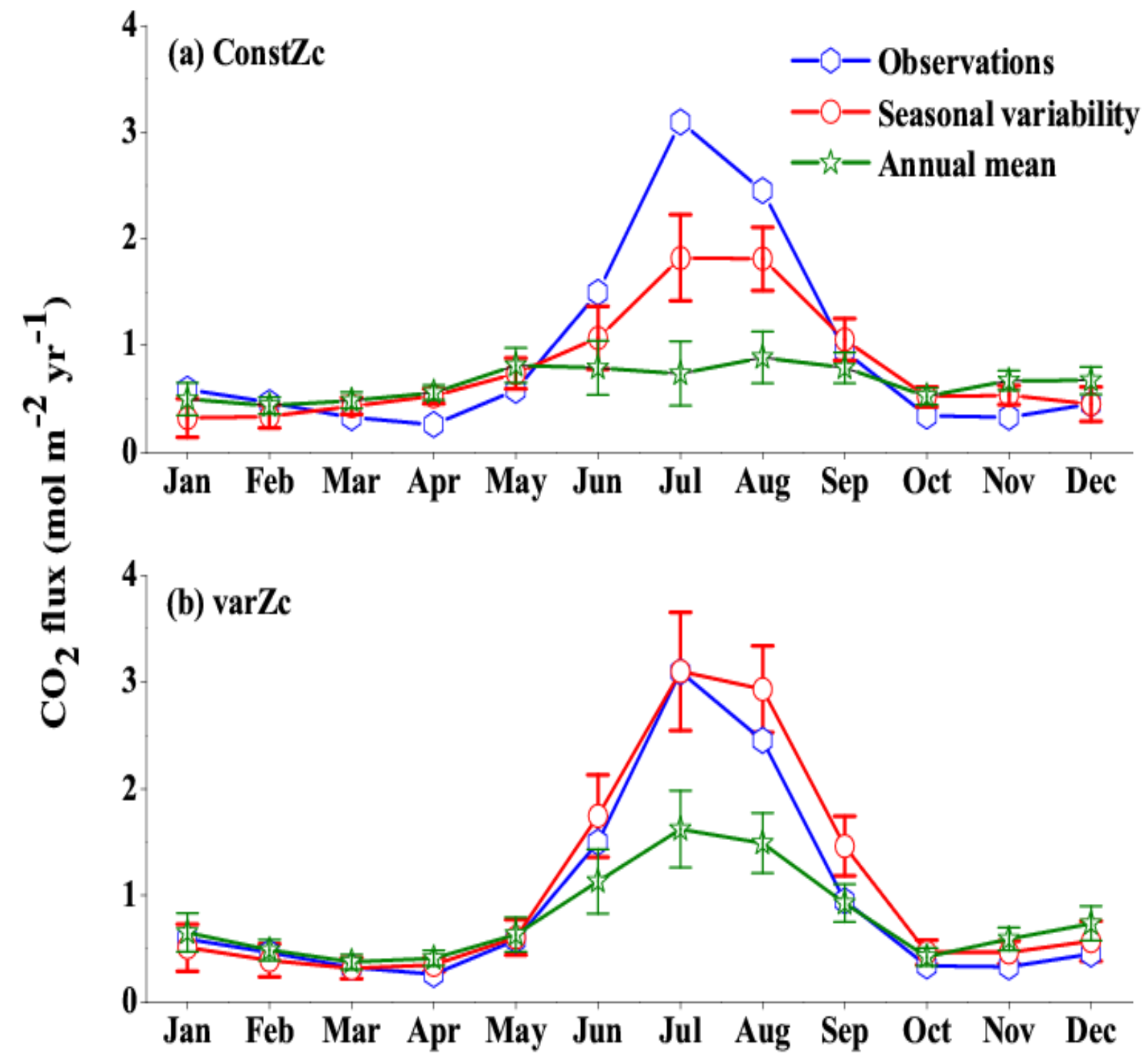

Figure S1: Response of $\mathrm{CO}_{2}$ flux from the model forced with annual mean currents over the WAS as climatology computed over 1990-2010. Error bar shows standard deviations of individual months over these years. (a) constZc and (b) varZc. Units are $\mathrm{mol} \mathrm{m}^{-2} \mathrm{yr}^{-1}$. Legends are same for both graphs. 


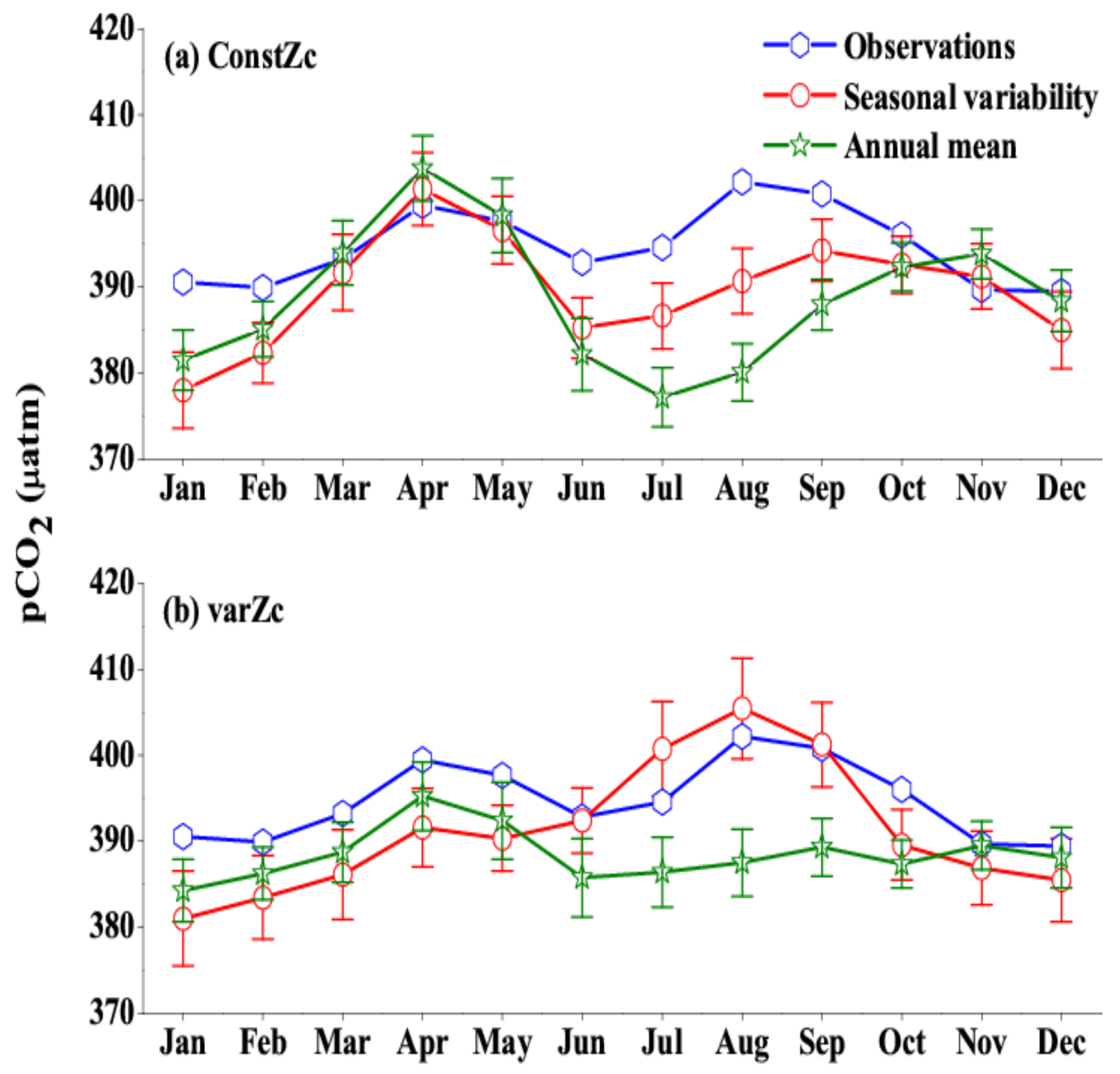

Figure $\mathrm{S} 2$ : Same as Figure $(\mathrm{S} 1)$, but for $\mathrm{pCO}_{2}$. Units are $\mu$ atm. 


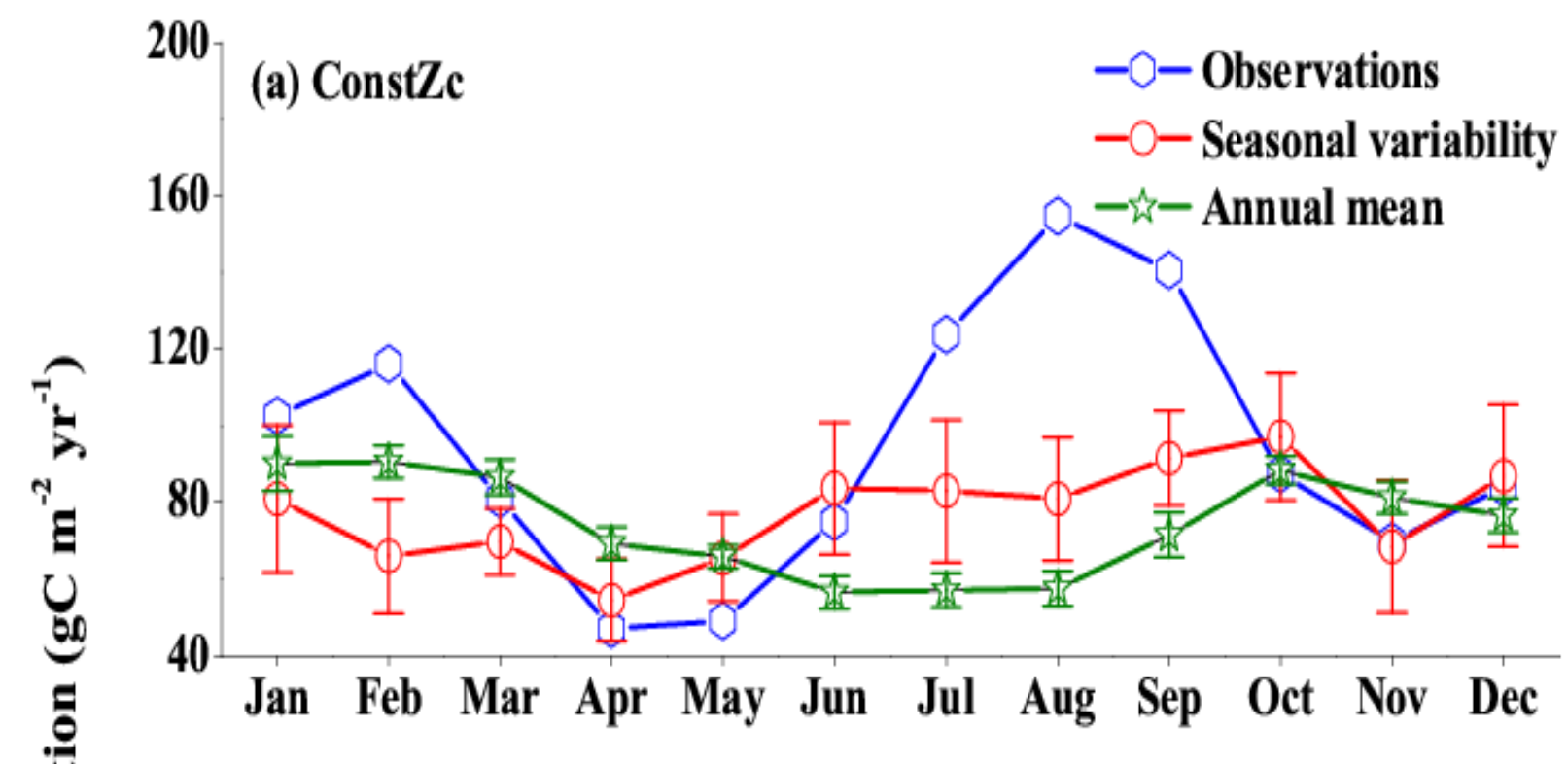

${ }^{200}$ (b) varZc

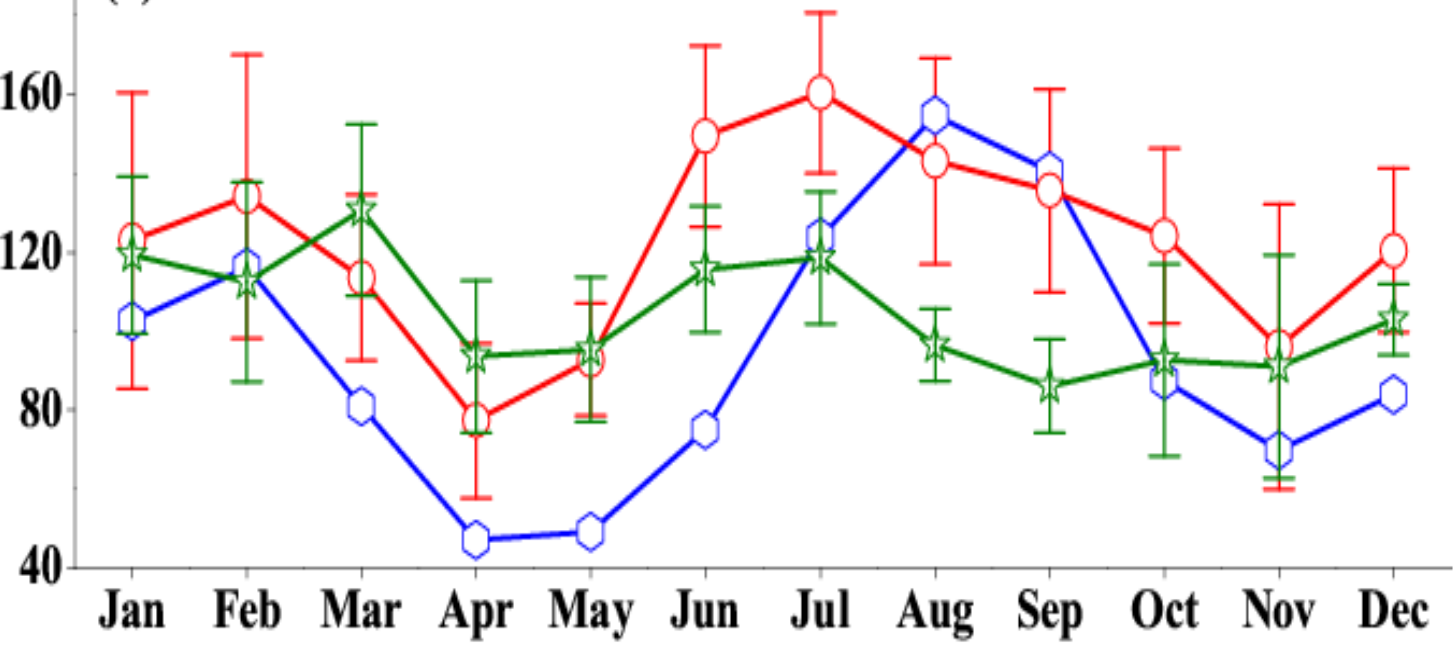

Figure S3: Response in the export production of the model forced with annual mean currents in the WAS as climatology computed over 1990-2010. Error bar shows standard deviations of individual months over these years. (a) constZc (b) varZc. Units are $\mathrm{g} \mathrm{C} \mathrm{m}^{-2} \mathrm{yr}^{-1}$. Legends are same for both graphs. 


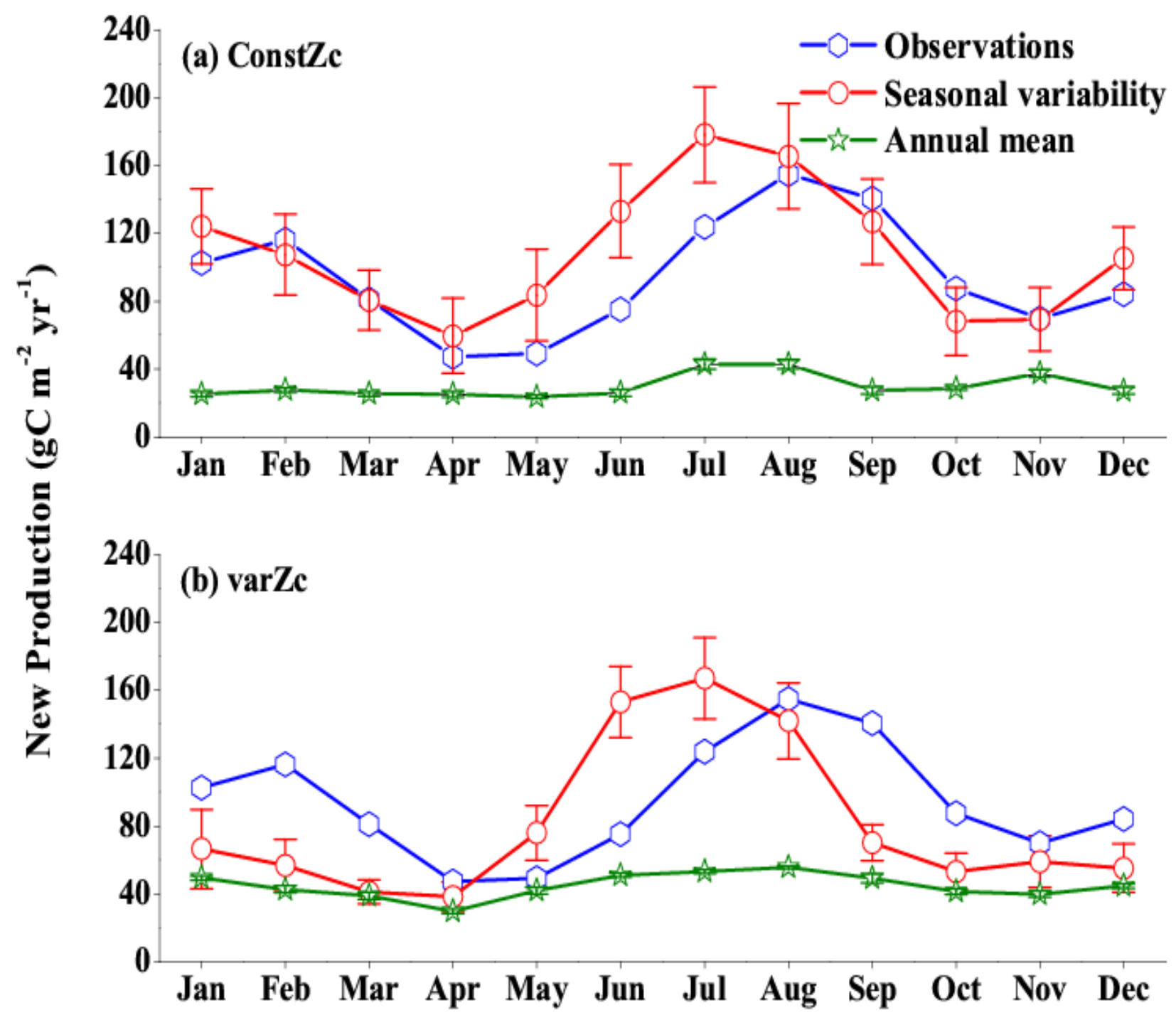

Figure S4: Same as Figure (S3), but for New Production. Units are $\mathrm{g} \mathrm{C} \mathrm{m}^{-2} \mathrm{yr}^{-1}$. 


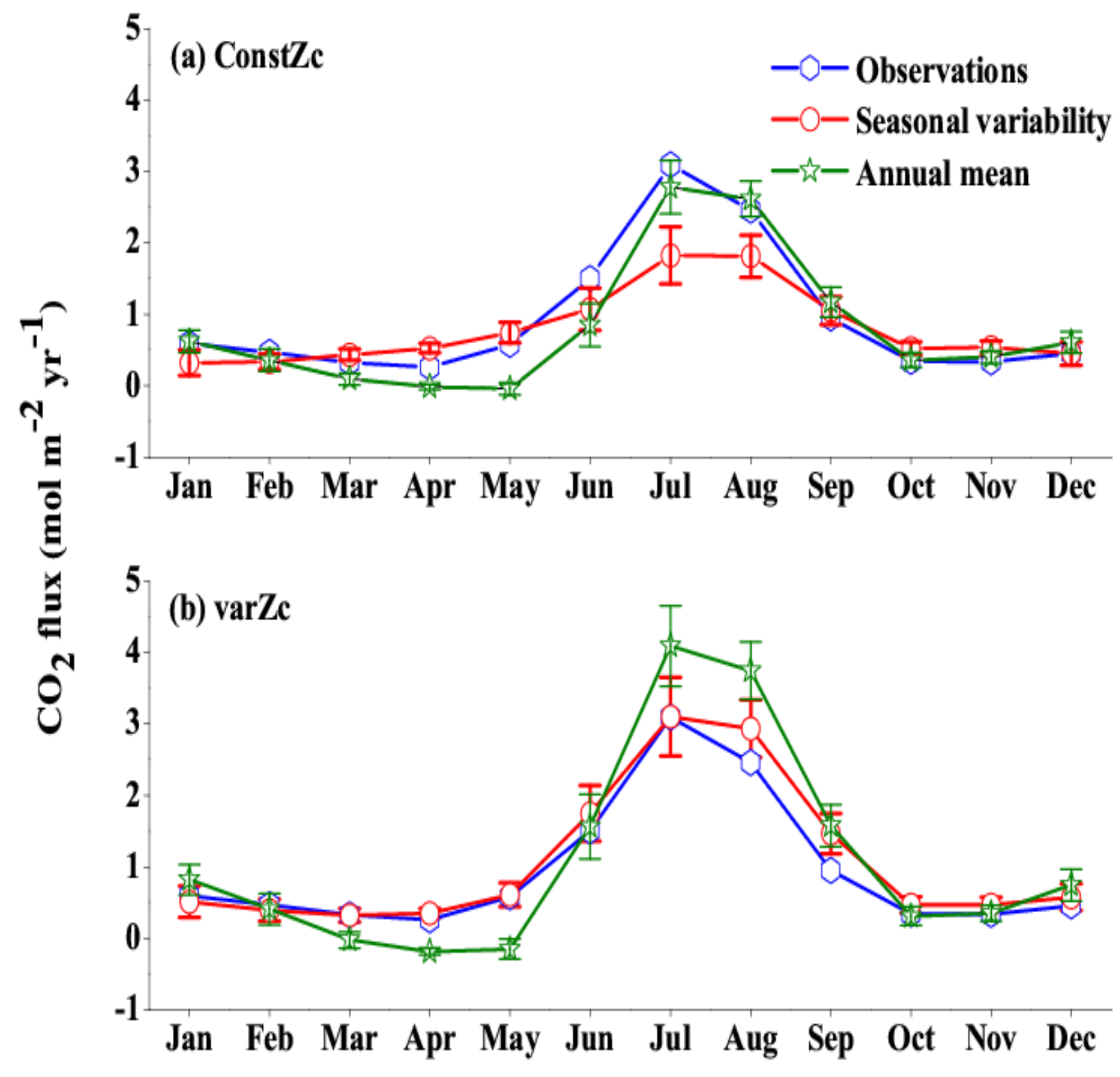

Figure S5: Response of $\mathrm{CO}_{2}$ flux from the model forced with annual mean SST over the WAS as climatology computed over 1990-2010. Error bar shows standard deviations of individual months over these years. (a) constZc and (b) varZc. Units are $\mathrm{mol} \mathrm{m}^{-2} \mathrm{yr}^{-1}$. Legends are same for both graphs. 


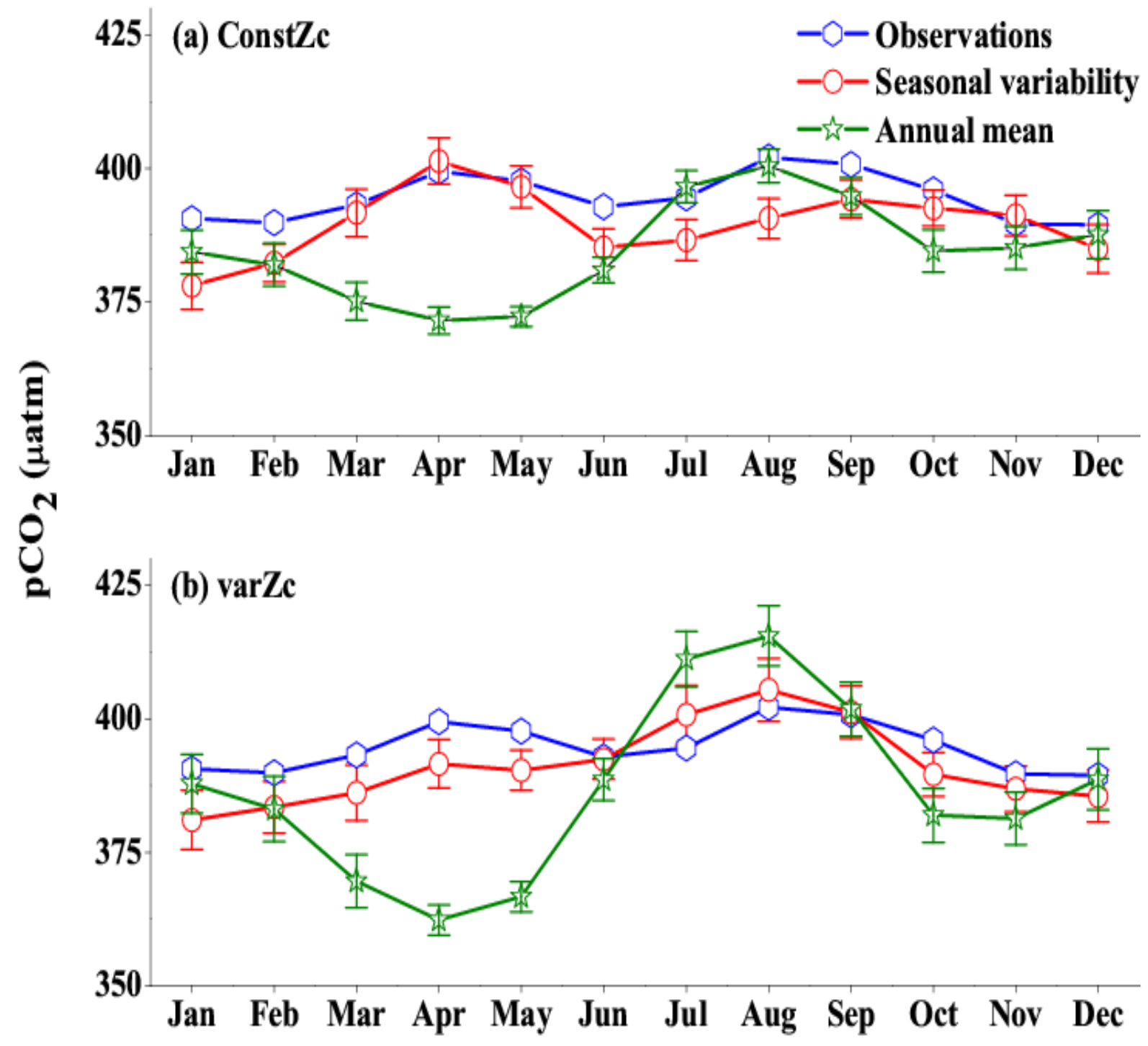

Figure S6: Same as Figure (S5), But for $\mathrm{pCO}_{2}$. Units are $\mu$ atm. 